Onishi et al.: The Effect of Metal Element Contents of Al Alloy Reflection Films on Sensitivity and Reliability of MagnetoOptical Disks

\title{
THE EFFECT OF METAL ELEMENT CONTENTS OF AI ALLOY REFLECTION FILMS ON SENSITIVITY AND RELIABILITY OF MAGNETO-OPTICAL DISKS
}

\author{
T. ONISHI, S. YAMAMOTO, K.YOSHIKAWA AND Y. KOGA*
}

\author{
Materials Research Laboratory, Kobe Steel, Ltd. \\ * New Business Division, Kobe Steel, Ltd. \\ 5-5, Takatsukadai, 1-chome, Nishi-ku, Kobe, HYOGO, 651-22, JAPAN
}

\begin{abstract}
We summarize the effect of alloying elements in $\mathrm{Al}$ alloy reflection films for quadri-layered MO disks. The addition of group 4A $7 \mathrm{~A}$ transition metal elements to $\mathrm{Al}$ films is the most effective to improve corrosion resistance and to reduce thermal conductivity. It is confirmed by practical tests that reliability and recording sensitivity of MO disks is extremely improved by using $\mathrm{Al}$-group $4 \mathrm{~A} \sim 7 \mathrm{~A}$ transition metal alloy reflection films.
\end{abstract}

KEY WORDS : MAGNETO-OPTICAL RECORDING, REFLECTION LAYER, Al ALLOY, CORROSION, THERMAL CONDUCTIVITY, SENSITIVITY, RELIABILITY

\section{INTRODUCTION}

Addition of metal element to Al reflection films is effective in improving the recording sensitivity and reliability of quadri-layered MO disks [1]. The recording sensitivity is mainly affected by the thermal conductivity of reflection films and the reliability is strongly affected by the corrosion behavior of reflection films.

In a previous paper, we discussed $\mathrm{Al}-\mathrm{Ti}$ and $\mathrm{Al}-\mathrm{Ta}$ alloys which exhibit a low solubility limit at equilibrium state [2]. They form a complete solid solution by sputter deposition, moreover they have high corrosion resistance and low thermal conductivity. In general, $\mathrm{Al}$ and transition metals (TM) do not form solid solutions at equilibrium state, and hence the same effect of $\mathrm{Ti}$ or $\mathrm{Ta}$ can be expected.

In this paper, we summarize the effects of other TM elements. We also examine the effect of $\mathrm{Mg}, \mathrm{Si}$ and $\mathrm{Cu}$, which are the usual alloying elements and which exhibit a high solubility limit to $\mathrm{Al}$, as compared with that of $\mathrm{TM}$ elements.

\section{EXPERIMENTAL PROCEDURE}

\section{Preparation and characterization of Al alloy films}

As shown in Table 2, 14 kinds of Al binary alloy systems were selected in this study. The 14 kinds of alloy elements are classified into three groups; group 4A 7A transition metal (TM4A 7A), group 8 transition metal (TM 8) and the usual alloying elements ( $\mathrm{Mg}, \mathrm{Si}$ and $\mathrm{Cu}$ ).

Pure $\mathrm{Al}$ films and the 14 kinds of $\mathrm{Al}$ alloy films were deposited by $\mathrm{DC}$ magnetron sputtering using vacuum melted alloy targets and composite targets. The vacuum pressure in the chamber prior to sputtering was less than 1x10-6 Torr. The metal element content of films was analyzed by induction coupled plasma emission spectroscopy (ICP) .

The microstructure of the sputtered films was investigated by X-ray diffraction (XRD), electron diffraction
(ED) and Transmission electron microscopy (TEM). Average grain size was calculated from TEM micrographs using the linear grain-boundary intercept method.

\section{Evaluation of corrosion properties}

Fig.1(a) shows the structure of samples used for environmental tests. Corrosion characteristics of the $\mathrm{Al}$ alloy reflection films were evaluated by measuring the reflectivity of the disk samples, before and after exposure to the corrosive environments at $105^{\circ} \mathrm{C}, 1.2 \mathrm{~atm}$ and $100 \%$ RH. Reflectivity was measured by the laser beam $(\lambda=780 \mathrm{~nm}, \phi 0.2 \mathrm{~mm})$ reflection method through PC substrates.

Anodic polarization of the $\mathrm{Al}$ alloy films was measured in $0.5 \% \mathrm{Na}_{2} \mathrm{SO}_{4}$ adjusted to $\mathrm{pH} 8.0$ with $\mathrm{H}_{2} \mathrm{SO}_{4}$ or $\mathrm{NaOH}$. Each specimen was prepared by depositing the $\mathrm{Al}$ alloy films onto a $10 \mathrm{~mm} \times 20 \mathrm{~mm}$ glass substrate, attaching a lead wire, and masking back and edge with resin coat. Further details of polarization measurements were reported in the previous paper [2].

Oxide layers on the polarized film surfaces were measured using a depth profile obtained from X-ray photoelectron spectroscopy (XPS). The surface structure of the passivation state was determined through wave analysis.

\section{Measurements of thermal conductivity}

The thermal conductivity of $\mathrm{Al}$ alloy films was measured with a thermal constant analyzer by the AC calorimetric method [3]. The specimen structure in these measurements is shown in Fig.1(b).

\section{Measurements of recording sensitivity}

The recording sensitivity of MO disks were evaluated using a MO disk tester (wavelength : 830nm). Fig.1(c) shows the cross-sectional structure of a $3.5 \mathrm{in}$.diam. disk employed in the dynamic recording test. The measurement conditions were shown in Table 1. 
Proceedings of Magneto-Optical Recording International Symposium '92, J. Magn. Soc. Jpn., Vol. 17, Supplement No. S1 (1993), pp. 106-109 (1) 1993 by The Magnetics Society of Japan

Table 1 Dynamic test conditions for MO disks.

\begin{tabular}{lr}
\hline Erase Power & $5.4 \sim 7.0 \mathrm{~mW}$ \\
Read-Out Power & $1.5 \mathrm{~mW}$ \\
Linear Velocity & $5.6 \mathrm{~m} / \mathrm{s}$ \\
Recording Frequency & $3.7 \mathrm{MHz}$ \\
Duty ratio & $33.3 \%$ \\
Erase Field & $250 \mathrm{Oe}$ \\
Write Field & $250 \mathrm{Oe}$ \\
\hline
\end{tabular}

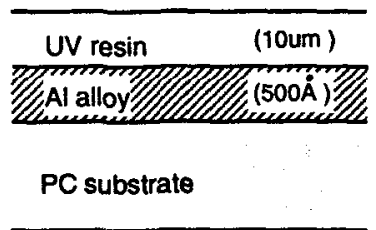

(a)

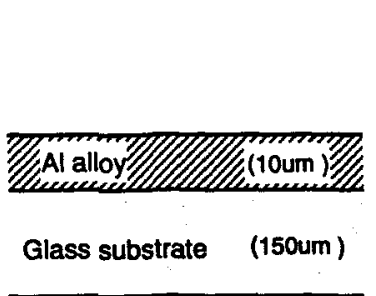

(b)

\begin{tabular}{|c|c|}
\hline UV resin & (10um) \\
\hline Al alloy & $(500 A)$ \\
\hline $\operatorname{SiN}$ & $(330 \AA)$ \\
\hline Tb-Fe-Co & $(250 \AA \dot{A})$ \\
\hline $\sin$ & $(980 \AA)$ \\
\hline \multicolumn{2}{|c|}{ Substrate (PC or Glass) } \\
\hline
\end{tabular}

(c)
Fig.1 The structure of disk samples.

\section{RESULTS AND DISCUSSION}

\section{Microstructure of Al alloy films}

The average grain size of pure $\mathrm{Al}$ films is approximately $1500 \AA$, and it is reduced with the addition of the metal elements. The average grain size of 14 kinds of Al-2 3at\%X alloy films (X: alloying elements) ranges from $350 \AA$ to $470 \AA$, and the difference in microstructure among these alloy films is not observed clearly. All of the Al alloy films examined in this study form complete solid solutions because ED patterns show only Al-fcc rings and no intermetallic compound is observed by XRD.

\section{Corrosion properties of Al alloy films}

Table 2 shows reflectivity reduction of the disk samples after the environmental tests. In these disk samples, the reflectivity reduction is the smallest for Al-TM 4A 7A alloy films as well as Al-Ti or Al-Ta alloy films. Reflectivity reduction of Al-TM 8 alloy films is slightly inferior to that of Al-TM 4A 7A alloy films, but the reflectivity reduction of Al-TM 8 alloy films is smaller than that of pure Al films. Among the other Al alloy films, the reflectivity reduction of $\mathrm{Al}-\mathrm{Cu}$ alloy films is relatively small, on the other hand, the reflectivity reduction of Al$\mathrm{Mg}$ alloy films is remarkably reduced. As mentioned above, all of the Al alloy films we tested form complete solid solutions. Therefore, corrosion reaction seems not to be accelerated by forming a local action cell.
Table 2 Reflectivity reduction of disk samples after environmental tests for 60 hours.

\begin{tabular}{lcccc}
\hline Element Group & $\begin{array}{c}\text { Content } \\
\text { (at\%) }\end{array}$ & $\begin{array}{c}\text { Solubility } \\
\text { limit(at\%) }\end{array}$ & $\begin{array}{c}\text { Reflectivity } \\
\text { reduction(\%) }\end{array}$ \\
\hline Pure Al & & & & 34.8 \\
$\mathrm{Mg}$ & $2 \mathrm{~A}$ & 3.20 & 17.8 & 63.0 \\
$\mathrm{Si}$ & $4 \mathrm{~B}$ & 1.97 & 1.5 & 10.1 \\
$\mathrm{Cu}$ & $1 \mathrm{~B}$ & 1.96 & 2.5 & 4.4 \\
$\mathrm{Ti}$ & $4 \mathrm{~A}$ & 2.65 & 0.75 & 1.4 \\
$\mathrm{Zr}$ & $4 \mathrm{~A}$ & 1.27 & 0.083 & 1.4 \\
$\mathrm{HI}$ & $4 \mathrm{~A}$ & 2.76 & 0.18 & 2.0 \\
$\mathrm{Nb}$ & $5 \mathrm{~A}$ & 2.69 & 0.064 & 1.5 \\
$\mathrm{Ta}$ & $5 \mathrm{~A}$ & 2.07 & 0.037 & 1.7 \\
$\mathrm{Cr}$ & $6 \mathrm{~A}$ & 3.09 & 0.40 & 1.7 \\
$\mathrm{Mo}$ & $6 \mathrm{~A}$ & 3.53 & 0.070 & 1.5 \\
$\mathrm{Mn}$ & $7 \mathrm{~A}$ & 2.73 & 0.90 & 1.5 \\
$\mathrm{Ni}$ & 8 & 3.62 & 0.023 & 2.9 \\
$\mathrm{Pd}$ & 8 & 2.93 & -0 & 15.2 \\
$\mathrm{Pt}$ & 8 & 2.20 & 1.0 & 24.9 \\
\hline
\end{tabular}

Fig. 2 shows the relationship between the reflectivity reduction and the current density at passive state (Ipassive) at $\mathrm{pH} 8.0$ for the $\mathrm{Al}$ alloy films.

A clear correlation between the reflectivity reduction and the Ipassive can be observed. Al-TM 4A 7A alloy films show excellent resistance to corrosion and their Log Ipassives are less than -6.1. Anodic polarization of each film is measured in neutral solution (pH8.0) because anodic polarization behavior measured at $\mathrm{pH} 8.0$ seems to reflect atmospheric corrosion. Each Al alloy film shows a smooth passive region owing to formation of an oxide film on the working electrode surface at anodic potential. Current density at passive state (Ipassive) corresponds to general corrosion rate, so Ipassive is determined by the anodic polarization curves. The excellent corrosion resistance of Al-TM $4 \mathrm{~A} \sim 7 \mathrm{~A}$ al-loy films seems to be attributed to the formation of rigid passivation films.

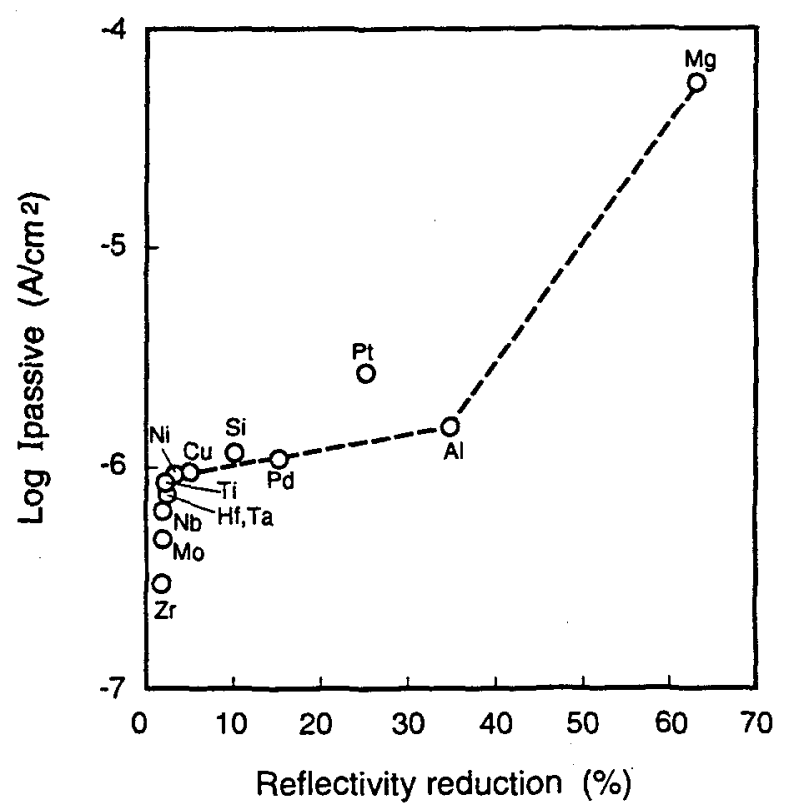

Fig.2 Relationship between the reflectivity reduction and Ipassive measured in $\mathrm{pH} 8.0$ for Al alloy films. 
Onishi et al.: The Effect of Metal Element Contents of Al Alloy Reflection Films on Sensitivity and Reliability of MagnetoOptical Disks

\section{Analysis of surface oxide films}

Fig. 3 shows the depth profiles of alloying elements in $\mathrm{Al}$ alloy films polarized at $\mathrm{pH} 8.0$. Al-TM 4A 7A alloy films and Al-TM 8 alloy films are covered with a surface oxide layer consisting exclusively of $\mathrm{Al} 3+$, no alloying element is detected. The surface $\mathrm{Al}_{2} \mathrm{O}_{3}$ layer seems to be formed with preferentially oxidized Al because alloying element enrichment under the surface oxide layer is not detected. The initial dissolution rate of $\mathrm{Al}$ in neutral solution is slow, therefore, the preferentially oxidized $\mathrm{Al}$ might form $\mathrm{Al}_{2} \mathrm{O}_{3}$ as surface oxidation layer without dissolving. The surface oxide structure on the $\mathrm{Al}$ alloy films polarized in neutral solution ( $\mathrm{pH} 8.0$ ) is approximately similar to that on $\mathrm{Al}$ alloy reflection films of the disk samples after the environmental tests. The excellent corrosion resistance of Al-TM 4A 7A alloy films might be attributed to the formation of a rigid passive film consisting of $\mathrm{Al}_{2} \mathrm{O}_{3}$.

The difference in reflectivity reduction between $\mathrm{Al}$ TM 4A 7A alloy films and Al-TM 8 alloy films cannot be explained completely form the view point of the difference of oxide film properties in relation to depth. It is reported that Al-TM 4A 7A alloy films have extraordinary pitting resistance [4 6]. These points should be taken into consideration.

In the case of $\mathrm{Al}-\mathrm{Mg}$ alloy films, oxide layer consists of preferentially oxidized $\mathrm{Mg}$, because $\mathrm{Mg}$ has a strong affinity for oxygen as compared with $\mathrm{Al}$. The poorest corrosion behavior of $\mathrm{Al}-\mathrm{Mg}$ alloy films is brought by $\mathrm{MgO}$ distribution in the surface oxide film because $\mathrm{MgO}$ does not form a stable passive film.

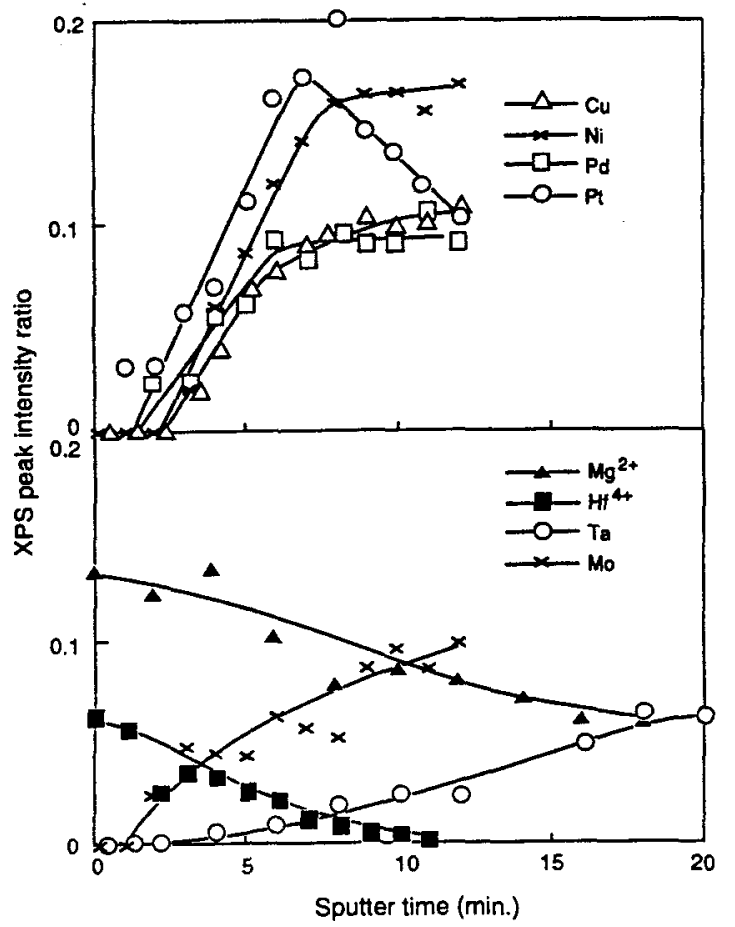

Fig.3 XPS depth profiles for alloy elements in Al binary alloy films polarized at $\mathrm{pH} 8.0$. The peak intensity ratio indicates the $[X]$ to $[A]$ ratio.

\section{Thermal conductivity of Al alloy films}

Fig. 4 shows the effect of alloy content on thermal conductivity for 7 kinds of $\mathrm{Al}$ alloy films. The effects of the elements are also classified into three groups; TM 4A $\sim 7 \mathrm{~A}, \mathrm{TM} 8$ and the usual alloying elements. The addition of TM 4A 7A elements is the most effective to reduce the thermal conductivity of $\mathrm{Al}$ films. Moreover, the addition of the usual alloying elements is not effective.

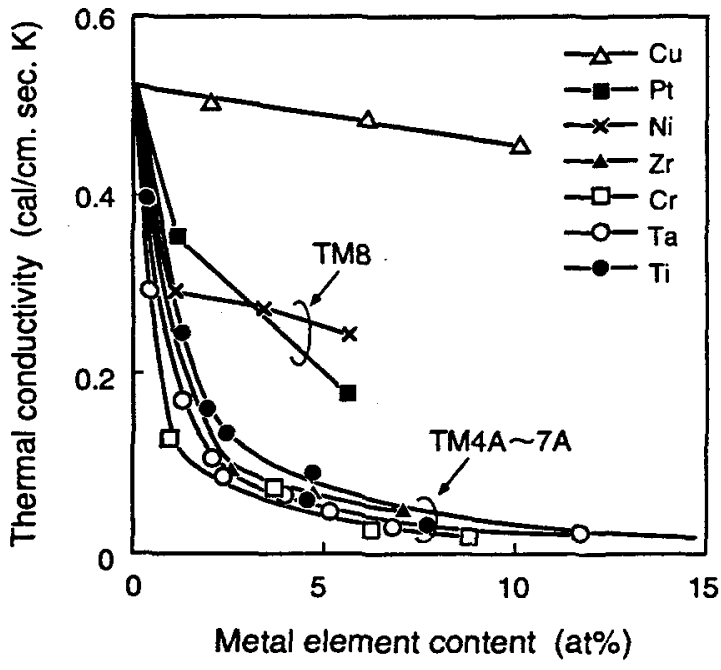

Fig.4 Thermal conductivity for Al alloy films.

The thermal conductivity reduction might be attributed to a scattering of conduction electrons because conduction electrons contribute greatly both to thermal conductivity and electrical resistivity in metallic materials. It can be estimated that in the case of Al-TM 4A 7A alloy films, $d$-band of TM 4A 7A overlap with the Fermi level of $\mathrm{Al}$ and conduction electrons of $\mathrm{Al}$ are scattered by the $d$-orbital. Non-equilibrium solid solution of TM 4A 7A elements brings this effect and remarkably reduce thermal conductivity. In the case of Al-TM 8 alloy films, the $d$-band does not overlap with the Fermi level of Al. Interaction between the $d$-band of TM elements and Fermi level of $\mathrm{Al}$ is calculated by the $\mathrm{DV}-\mathrm{X} \alpha$ method, which is one of the molecular orbital methods, from the view point of investigation of electrical resistivity [7]. There is a close relationship between thermal conductivity and electrical resistivity of the $\mathrm{Al}$ alloy films as shown by comparison of Figures 4 and 5 . The considerable reduction of thermal conductivity in Al-TM 4A 7A alloy films systems to be due to the electronic structure change of $\mathrm{Al}$ by the occurrence of virtual bound state in $\alpha$-Al crystals.

Lattice distortions, lattice defects and grain boundaries seem to be one of the origins of the considerable reduction of thermal conductivity in Al-TM 4A 7A alloy films. But in this study, grain boundary scattering is not a fundamental factor on the thermal conductivity reduction because there is no difference in average grain size among the $\mathrm{Al}$ alloy films. 


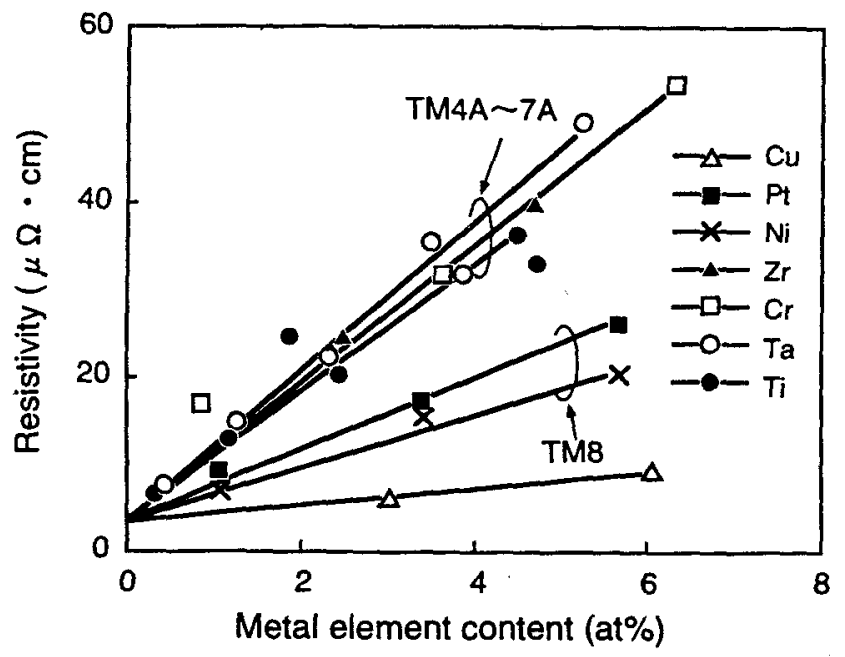

Fig.5 Electric resistivity for Al alloy films. Resistivity of specimen having a thickness of 2000A was measured by four-point probe method.

\section{Recording sensitivity of $M O$ disks}

Fig.6 shows the relationship between recording laser power and Carrier to Noise Ratio (CNR) for MO disks with pure $\mathrm{Al}$ and the $\mathrm{Al}$ alloy reflection films. There is no difference in CNR between pure $\mathrm{Al}$ and the $\mathrm{Al}$ alloy reflection film. The optimum writing power of high sensitive MO disks is lower than that of usual MO disks. Therefore the top laser power to bottom laser power ratio should be reduced. It can be notified that CNR is not influenced by the laser power ratio from these results.

Fig. 7 shows the sensitivity of MO disks containing 7 kinds of $\mathrm{Al}$ alloy reflection films. A clear correlation between the sensitivity of MO disks and the thermal conductivity of reflection films is indicated. MO disks containing the low thermal conductivity reflection layers, that is, Al-TM 4A 7A alloy reflection films, have high recording sensitivity without lowering CNR.

The actual thickness of reflection layer of MO disks, about $500 \AA$ thickness, is thinner than that of specimen used for thermal conductivity measurement. Further thermal conductivity reduction can be expected in actu-

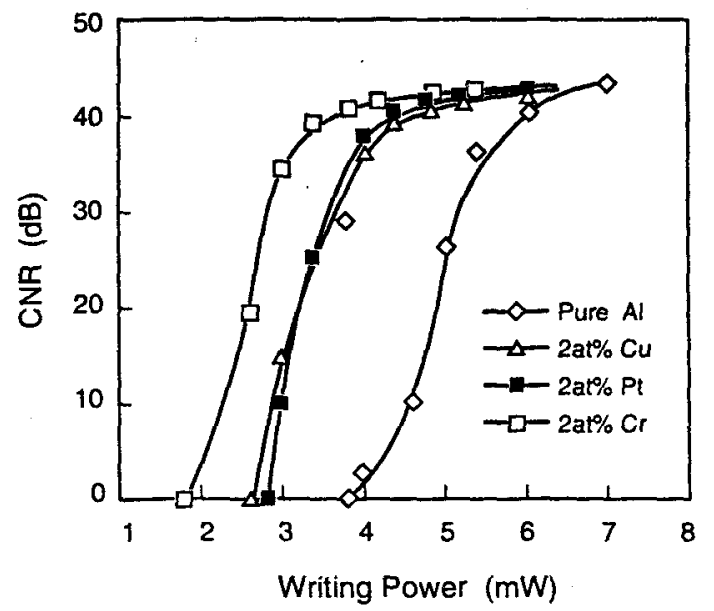

Fig.6 Relationship between the recording laser power and CNR for each of MO disks.

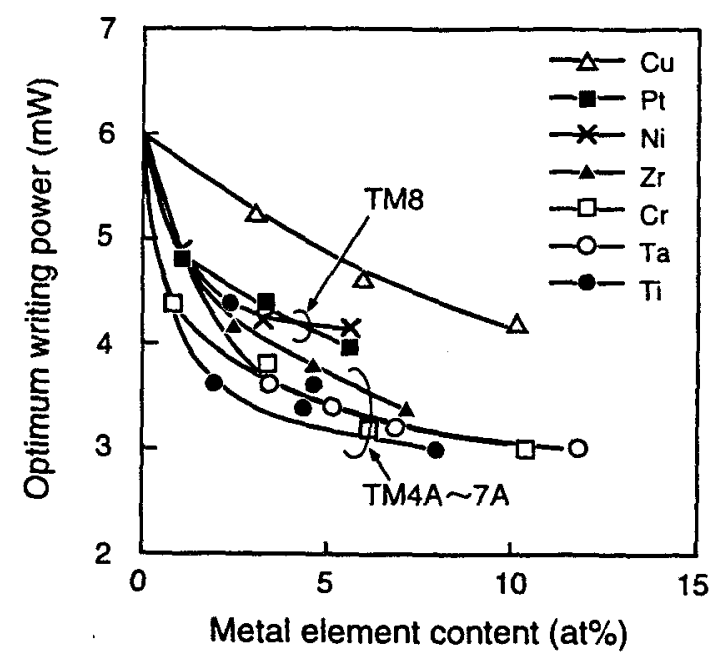

Fig.7 Relationship between metal element contents of $\mathrm{Al}$ alloy reflection films and optimum writing power of $\mathrm{MO}$ disks.

al reflection layers of MO disks because, in the case of thinner films, the conduction electron scattering at the film surface becomes a dominant factor in thermal conductivity [8]. From the results shown in Fig.5 and Fig.7, however, the same tendency for the thermal conductivity reduction was observed.

\section{CONCLUSION}

The addition of TM 4A 7A elements to $\mathrm{Al}$ is the most effective upon the improvement of corrosion resistance and the decrement of thermal conductivity. The excellent corrosion resistance of Al-TM 4A 7A alloy films might be attributed to the formation of rigid passive films. The considerable reduction of the thermal conductivity in Al-TM 4A 7A alloy films are realized by scattering of conduction electron of $\mathrm{Al}$ by the $d$-band of the TM alloys. It was confirmed by practical tests that the corrosion resistance and the thermal conductivity of reflection films strongly reflect the reliability and the recording sensitivity of MO disks. Al-TM alloy films are the most suitable as reflection films for MO disks.

\section{REFERENCES}

[1] T. Kawano, Mitsubishi Kasei R \& D Review, 4(2), 1 (1990)

[2] T. Onishi et al., the Journal of the Magnetic Society of Japan, 15(S1), 373(1991)

[3] I. Hatta et al., Rev. Sci. Instrum., 56, 1643(1989)

[4] W. C. Moshier et al., J. Electrochem. Soc., 134(11), 2677(1987)

[5] G. S. Frankel et al., J. Electrochem. Soc., 136(4), 1243(1989)

[6] B. A. Shaw et al., J. Electrochem. Soc., 137(4), 1317 (1990)

[7] J. Deutz et al., J. Phys. F, 11, 1787(1981)

[8] D. Raasch et al., J. Magn. Magn. Mater., 93, 365 (1991) 